\title{
Assessment of an optimized dog-culling program in the dynamics of canine Leishmania transmission
}

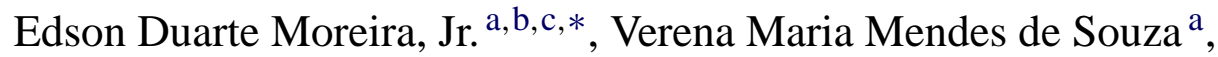 \\ Meera Sreenivasan $^{\mathrm{d}}$, Eliane Góes Nascimento ${ }^{\mathrm{e}}$, \\ Lain Pontes de Carvalho ${ }^{\text {a }}$ \\ a Centro de Pesquisas Gonçalo Moniz, Fundação Oswaldo Cruz, Rua Waldemar Falcão 121, \\ Salvador, Bahia CEP 40295-001, Brazil \\ b Núcleo de Apoio à Pesquisa, Associação Obras Sociais Irmã Dulce, Av. Bonfim 161, \\ Salvador, Bahia 40420-000, Brazil \\ ${ }^{c}$ Diretoria Científica, Hospital São Rafael, Av. São Rafael 2152, Salvador, Bahia 41256-900, Brazil \\ d Division of Epidemiology and Public Health Biology, School of Public Health, \\ University of California at Berkeley, 140 Warren Hall, Berkeley, CA 94720, USA \\ e Centro de Referência em Doenças Endêmicas Pirajá da Silva, Secretaria da Saúde do Estado da Bahia, \\ Rua 3 s/n, Casas Populares, Jequié, Bahia 45200, Brazil
}

Received 1 May 2004; accepted 21 May 2004

\begin{abstract}
In Brazil, zoonotic visceral leishmaniasis (ZVL) control programs based on the mass elimination of seropositive dogs have failed to reduce the number of leishmaniasis cases. However, these programs have been done under sub-optimal conditions. We studied a cohort of dogs in an urban area in Brazil to determine, whether a dog-culling program optimized with: (i) replacement of a relatively low-sensitivity indirect immune-fluorescent test on blood eluate by a more sensitive enzyme-linked immunosorbent assay on serum blood samples; (ii) shortening of the time interval from serodiagnosis to removal of dogs; (iii) screening a high proportion of the dog population could reduce the incidence of canine Leishmania infection (CLI). The study ran from December 1997 to July 2000, with four follow-up assessments performed at approximately 8-month intervals. All dogs seropositive for anti-Leishmania antibodies were promptly eliminated. A large number of new dogs immigrated to the study area throughout the study period. They comprised $43.8-49.8 \%$ of the cohort at each follow-up assessment, and upto 15\% of them already had Leishmania infection. Overall, 42 news cases of CLI were identified, for a crude incidence rate of 11.8 cases per 100 dog-years (95\% CI 8.6-15.6). In the first, second, third and fourth follow-up assessments the incidence rates were 8.2 (95\% CI 3.0-17.9), 12.2 (95\% CI 6.3-21.2), 16.4 (95\% CI 8.5-28.6) and 13.6

\footnotetext{
* Corresponding author. Present address: Centro de Pesquisas Gonçalo Moniz, Fundação Oswaldo Cruz, Rua Waldemar Falcão 121, Salvador, Bahia CEP 40295-001, Brazil. Tel.: +55 71356 8781x243;

fax: +55713562155 .

E-mail address: edson@ @pqgm.fiocruz.br (E.D. Moreira Jr.).
} 
(95\% CI 7.1-23.8), respectively. There was no statistically significant change in these rates throughout the study period. Our results suggest that dog-culling programs do not reduce the incidence of CLI, even with an optimized intervention. Possible reasons for this failure include: currently available serologic methods lack sufficient sensitivity and/or specificity to accurately identify all infected dogs warranting removal in order to prevent Leishmania transmission; destroyed dogs are immediately replaced by susceptible puppies, and quite often, by already infected dogs; and other reservoirs may be involved in maintaining canine infection. Further efforts on ZVL control should be directed to developing new strategies or to testing control methods already in place with properly designed trials. (C) 2004 Elsevier B.V. All rights reserved.

Keywords: Incidence; Canine visceral leishmaniasis; Leishmania infection; Control; Prevention; Cohort study

\section{Introduction}

Canine visceral leishmaniasis (CVL) was first documented in Tunisia by Nicolle and Comte (1908). In Latin America, CVL is caused by infection with Leishmania chagasi (Maurício et al., 1999) and is usually transmitted by the sandfly Lutzomyia longipalpis. In Brazil, the prevalence of canine infection in endemic regions ranges from 1 to $36 \%$, and reaches upto 67\% in certain areas (Coutinho et al., 1985; Paranhos-Silva et al., 1996; França-Silva et al., 2003).

Domestic dogs are believed to be the main vertebrate reservoir for zoonotic visceral leishmaniasis (ZVL), a major public health problem in tropical America. Over the past 5 years, the Brazilian Ministry of Health reported a national annual average of 3500 cases, and ZVL is acknowledged as an important emerging disease. In developed areas such as the Mediterranean, it is both a medical and a veterinary problem, whereas in developing areas it is primarily a medical problem (Tesh, 1995).

Control programs in Brazil have focused on the mass elimination of seropositive dogs. However, Brazilian National Health data for past decades show that widespread culling of seropositive dogs does not reduce the number of human cases (Vieira and Coelho, 1998). This has prompted a reassessment of the dog control policy in Brazil (Costa and Vieira, 2001). Although, some studies suggest that dog elimination control programs are ineffective (Dietze et al., 1997; Courtenay et al., 2002), it is questionable, whether limitations of the intervention methods used in these studies, such as the use of low-sensitivity screening assays and delays in removing seropositive dogs, might have been responsible for the apparent failure of this control strategy. In order to contribute to solving this controversy, we conducted a prospective cohort study to determine the impact of an optimized dog-culling program on the incidence of canine Leishmania infection (CLI).

\section{Methods}

\subsection{Study area}

The study site is located in the city of Jequié, Bahia, Brazil, an endemic area for CVL, with a population of 1,47,202 inhabitants (Instituto Brasileiro de Geografia e Estatística, 2001). We selected a borough (São Judas Tadeu) with 376 households and 1873 inhabitants 
to perform the study, because its CLI prevalence was high (31\%) and its isolated location on the periphery of the city made boundaries easy to demarcate (Paranhos-Silva et al., 1996; Moreira et al., 2003).

\subsection{Study design}

In the baseline assessment (December 1997), we conducted a population-based canine survey of all domiciled dogs, aged 6 months or older, living in the study area. All seropositive dogs were painlessly eliminated, following guidelines of the Brazilian National Foundation of Health, and the remaining seronegative dogs were included in our initial cohort for follow-up. The study ran from December 1997 to July 2000, during which time four follow-up assessments were performed, at approximately 8-month intervals. This was a dynamic cohort since, at each follow-up survey, new dogs that had immigrated to the study area were tested for Leishmania infection, and if seronegative, included in the cohort. Otherwise, these dogs and those in the cohort that seroconverted were killed within 14 days of the survey.

\subsection{Data and blood collection}

Information on age, sex, breed, and other characteristics was gathered using a standardized questionnaire, administered to the consenting owners of each animal by trained and certified interviewers. All data were collected at study entry and updated at each follow-up assessment. Blood samples were collected by venipuncture from all animals available at each survey.

\subsection{Serology}

An enzyme-linked immunosorbent assay (ELISA), which had been previously validated and described elsewhere (Paranhos-Silva et al., 1996), was used to determine the presence of anti-Leishmania antibodies. Positive and negative control sera were included in every assay. All sera were tested in duplicate and those yielding positive results were retested at least once.

\subsection{Statistical analysis}

Incidence density rates for CLI were calculated as the number of new infections (defined as seroconversions at follow-up) divided by the number of dog-years of follow-up. Dog-years were defined as the number of years between the initial and the last assessment for each dog at risk for Leishmania infection (dogs with only one assessment did not contribute any time to the total of dog-years). The overall incidence rate as well as the specific rates for each follow-up interval were estimated with respective $95 \%$ confidence intervals (CI).

\section{Results}

The mean age of the 447 dogs at study entry was 1.7 years (range $0.5-14$ ). The cohort was largely comprised of young, short fur mongrel dogs. Selected characteristics of dogs in 
Table 1

Selected characteristics of dogs in the study cohort, Jequié, Bahia, Brazil, 1997-2000

\begin{tabular}{|c|c|c|c|}
\hline Characteristics & Cohort $(n=447)$ & Analysis sample $(n=245)$ & Lost to follow-up $(n=202)$ \\
\hline \multicolumn{4}{|l|}{ Age (years) } \\
\hline$\leq 1$ & $256(57.3 \%)$ & $127(51.9 \%)$ & $129(63.9 \%)$ \\
\hline 2 & $85(19.0 \%)$ & $55(22.4 \%)$ & $30(14.8 \%)$ \\
\hline 3 & $54(12.1 \%)$ & $35(14.3 \%)$ & $19(9.4 \%)$ \\
\hline$\geq 4$ & $52(11.6 \%)$ & $28(11.4 \%)$ & $24(11.9 \%)$ \\
\hline \multicolumn{4}{|l|}{ Gender } \\
\hline Male & $237(52.8 \%)$ & $135(55.1 \%)$ & $102(50.5 \%)$ \\
\hline Female & $210(47.2 \%)$ & $110(44.9 \%)$ & $100(49.5 \%)$ \\
\hline \multicolumn{4}{|l|}{ Breed } \\
\hline Mixed & $404(90.4 \%)$ & $225(91.9 \%)$ & $179(88.6 \%)$ \\
\hline Pure & $43(9.7 \%)$ & $20(8.2 \%)$ & $23(11.4 \%)$ \\
\hline \multicolumn{4}{|l|}{ Fur length } \\
\hline Long & $41(9.2 \%)$ & $14(5.7 \%)$ & $27(13.4 \%)$ \\
\hline Short & $406(90.8 \%)$ & $231(94.3 \%)$ & $175(86.6 \%)$ \\
\hline \multicolumn{4}{|l|}{ Degree of confinement } \\
\hline Limited to the backyard & $178(39.8 \%)$ & $78(31.8 \%)$ & $100(49.5 \%)$ \\
\hline Free to roam & $269(60.2 \%)$ & $167(68.2 \%)$ & $102(50.5 \%)$ \\
\hline
\end{tabular}

the whole cohort, in the analysis sample (comprised by dogs with at least two assessments), and in the lost to follow-up group are shown in Table 1. This allows for comparison of variable distributions from cohort entry to follow-up, and assessment of how attrition and exclusion of selected groups might have affected the sample remaining in the cohort. Dogs in the analysis sample were similar to those lost to follow-up with regard to the distribution of age, gender, and breed. Yet, animals lost to follow-up were more likely to have long fur and to be raised confined in the backyard than dogs in the analysis sample (Table 1). The average lost to follow-up rate of the four follow-up surveys was $35.8 \%$, ranging from 29.8 to $47.9 \%$ (Table 2). Of the 202 dogs excluded, 129 (63.9\%) moved out of the area, 35 $(17.3 \%)$ died, $1(0.5 \%)$ had owner's consent withdrawn, and on $37(18.3 \%)$ there was no information available. A large number of new dogs immigrated to the study area throughout the study period. They comprised $43.8-49.8 \%$ of the cohort at each follow-up assessment

Table 2

Population dynamics in a cohort of dogs in Jequié, Brazil, 1997-2000

\begin{tabular}{lllll}
\hline Assessment & $\begin{array}{l}\text { Number of } \\
\text { dogs in the } \\
\text { cohort }\end{array}$ & $\begin{array}{l}\text { Proportion (\%) } \\
\text { of dogs lost to } \\
\text { follow-up }\end{array}$ & $\begin{array}{l}\text { Proportion (\%) } \\
\text { of immigrant } \\
\text { dogs }\end{array}$ & $\begin{array}{l}\text { Seropositivity (\%) } \\
\text { among immigrant } \\
\text { dogs }\end{array}$ \\
\hline Baseline (December 1997) & 181 & - & - & - \\
Follow-up 1 (July 1998) & 223 & $29.8(54 / 181)$ & $49.8(111 / 223)$ & $13.5(15 / 111)$ \\
Follow-up 2 (March 1999) & 231 & $34.1(74 / 217)$ & $43.7(101 / 231)$ & $12.9(13 / 101)$ \\
Follow-up 3 (November 1999) & 195 & $47.9(106 / 219)$ & $49.2(96 / 195)$ & $14.6(14 / 96)$ \\
Follow-up 4 (July 2000) & 239 & $31.7(58 / 183)$ & $49.8(119 / 239)$ & $4.2(5 / 119)$ \\
\hline
\end{tabular}


Table 3

Incidence density rates of canine Leishmania infection in a cohort of dogs in Jequié, Brazil, 1997-2000

\begin{tabular}{lllll}
\hline Assessment & $\begin{array}{l}\text { Number of dogs in } \\
\text { the cohort }\end{array}$ & $\begin{array}{l}\text { Number of } \\
\text { incident cases }\end{array}$ & Dog-years & $\begin{array}{l}\text { Incidence/100 } \\
\text { dog-years (95\% CI) }\end{array}$ \\
\hline Baseline (December 1997) & 181 & - & - & - \\
Follow-up 1 (July 1998) & 223 & 6 & 72.8 & $8.2(3.0-17.9)$ \\
Follow-up 2 (March 1999) & 231 & 12 & 98.7 & $12.2(6.3-21.2)$ \\
Follow-up 3 (Novemer 1999) & 195 & 12 & 73.3 & $16.4(8.5-28.6)$ \\
Follow-up 4 (July 2000) & 239 & 12 & 88.0 & $13.6(7.1-23.8)$ \\
\hline
\end{tabular}

(Table 2). Upto $15 \%$ of the dogs immigrating to the study site were already seropositive for Leishmania antibodies.

The average follow-up time was 1.5 years (range 0.6-2.6). Overall, 42 new cases of CLI were identified in 357.5 dog-years of follow-up, for a crude incidence rate of 11.8 cases per 100 dog-years (95\% CI 8.6-15.6). The specific incidence rates for each follow-up interval are shown in Table 3. Although, the incidence rate of CLI in the first follow-up interval seemed smaller than the rates in the following assessments, there was no statistically significant change in the incidence of CLI throughout the study period.

\section{Discussion}

The overall incidence rate of CLI in our population (11.8 cases/100 dog-years) was similar to the annual incidence found on the Isle of Elba (12.4\%) and in western Liguria (11.2\%), both in Italy (Gradoni et al., 1988; Zaffaroni et al., 1999), but it was higher than previously reported estimates in the same area (6.6 cases/100 dog-years) (Paranhos-Silva et al., 1998), and elsewhere in Brazil (6.4 cases/100 dog-years) (França-Silva et al., 2003). Despite strict adherence to a well-defined protocol to screen and eliminate seropositive dogs promptly, the rates of Leishmania infection have not decreased throughout the study period, but instead remained stable. Thus, our findings do not support the removal of serologically positive dogs as a strategy to reduce the incidence of CLI, and consequently, argue against using this measure in ZVL control programs. Some earlier studies have also questioned the effectiveness of killing infected dogs (Alencar et al., 1974; Dye, 1996; Dietze et al., 1997; Courtenay et al., 2002). Nevertheless, Ashford et al. (1998) found that culling dogs was partially successful, and Palatnik-de-Sousa et al. (2001) reported that the removal of seropositive dogs led to a significant reduction of the incidence of canine and human Leishmania infection. However, even when such conclusions are drawn from controlled trials, the methodological shortcomings in the study design, analysis or in the conduction of the interventions invariably impair the interpretation and validity of these data.

Numerous obstacles exist that prevent dog-culling programs from achieving good disease control. Limitations including long intervals between serodiagnosis and dog removal, lower than ideal sensitivity and/or specificity of currently available screening tests, and owner's unwillingness to give up seropositive dogs are difficult to overcome, further complicating these interventions. Moreover, a pivotal reason for the failure of dog-culling to control 
CVL is the lack of a reliable test to identify infectious rather than infected dogs. This is an important distinction because a proportion of infected dogs might never become infectious, thus their removal would be actually counterproductive, since they may be replaced by susceptible dogs that do become infectious. In addition, other reservoirs may be involved in maintaining canine infections (Gradoni et al., 1983; Corredor et al., 1989), and interventions aimed at an individual species may be less effective when multiple reservoirs are involved in transmission.

We found a high turn-over of dogs in the study area, where destroyed dogs were immediately replaced by susceptible puppies. Furthermore, a fraction of the dogs immigrating to the study site was already infected. This combination would negatively affect the efforts aimed at reducing the prevalence of CLI in the dog population and might have also played an important role in the failure of the dog-culling program. Indeed, in this scenario, modeling theory indicates that a high proportion of infectious dogs should be killed in order to produce a marked reduction in disease transmission (Dye, 1996). It might be the case, that such an effective performance cannot be accomplished even with an optimized intervention such as the one described herein. Thus, this advises against recommending a dog-culling intervention as a nation-wide ZVL control strategy, since such programs are too laborious and therefore, likely to be less thorough and effective when applied in a large-scale.

\subsection{Methodological merits and limitations}

The main strength of this study was its prospective design that allowed for proper calculation of CLI incidence rates at short time intervals. In addition, the study area was surveyed thoroughly and we achieved a high coverage of the dog population. The losses to follow-up were mainly due to emigration from the study area and are likely to be non-differential, thus it is improbable that selection bias might have distorted our results. Also, all seroconverted dogs were killed promptly, avoiding long intervals between serodiagnosis and dog removal.

The main limitation was that we could not establish a control site. Ethical considerations precluded that we surveyed dogs for anti-Leishmania antibodies in a control area without removing the seropositive animals. The inclusion of a control group would have supplied the expected rate of CVL transmission in the absence of the intervention. Nonetheless, the lack of a comparison group should not compromise the interpretation of our findings, since the infection rates remained stable throughout the study suggesting that the intervention has not prevented Leishmania transmission in the study area.

\subsection{Conclusions and public health implications}

We conclude that dog-culling programs do not reduce the incidence of CLI, even when the intervention is optimized with: (i) replacement of a relatively low-sensitivity indirect immune-fluorescent test on blood eluate by a more sensitive ELISA test on serum blood samples; (ii) shortening of the time interval from serodiagnosis to removal of dogs; (iii) screening a high proportion of the dog population. Possible reasons for this failure include: currently available serologic methods lack sufficient sensitivity and/or specificity to accurately identify all infected dogs warranting removal in order to prevent Leishmania transmission; destroyed dogs are immediately replaced by susceptible puppies, and quite 
often, by already infected dogs; and other reservoirs may be involved in maintaining canine infection. Further efforts on ZVL control should be directed towards developing new strategies, such as a canine or human vaccine against Leishmania, and towards conducting properly designed ZVL control trials to evaluate the effectiveness of other available control approaches, such as residual insecticide spraying or the use of insecticidal dog collars. The fundamental role of human susceptibility to leishmaniasis, with focus on the alleviation of malnutrition, also remains to be investigated. Of utmost importance, cost-effectiveness studies should always be performed before broad control measures are recommended and adopted nationwide.

\section{Acknowledgements}

This research was funded by a grant (PV-98935) from the Centro de Apoio ao Desenvolvimento Científico e Tecnológico (CADCT), a grant (52.1122/98) from the Conselho Nacional de Desenvolvimento Científico e Tecnológico (CNPq), and by the PRONEX (MCT). We thank Dr. Moacyr Paranhos-Silva, Dr. Paulo P. Aguiar, Dr. Maria F.A. Cruz, Mr. Carlos F.L. Lobo, Mr. Antônio de Almeida Filho, Mrs. Elivani S. de Jesus and Mrs. Rita C.J. Santos for helping with data collection and Dr. Washington L.C. Santos for his helpful comments. We are also grateful to all health care workers and technicians at the PIEJ Clinic in Jequié.

\section{References}

Alencar, J.E., Almeida, Y.M., e Silva, Z.F., Paiva, A.S., da Fonseca, M.F., 1974. Current aspects of kala-azar in Ceara. Rev. Bras. Malariol Doencas Trop. 26, 27-53.

Ashford, D.A., David, J.R., Freire, M., David, R., Sherlock, I., Eulalio, M.C., Sampaio, D.P., Badaro, R., 1998. Studies on control of visceral leishmaniasis: impact of dog control on canine and human visceral leishmaniasis in Jacobina, Bahia, Brazil. Am. J. Trop. Med. Hyg. 59, 53-57.

Corredor, A., Gallego, J.F., Tesh, R.B., Morales, A., Carrasquilla, C.F., Young, D.G., Kreutzer, R.D., Boshell, J., Palau, M.T., Caceres, E., Pelaez, D., 1989. Epidemiology of visceral leishmaniasis in Colombia. Am. J. Trop. Med. Hyg. 40 (5), 480-486.

Costa, C.H., Vieira, J.B., 2001. Changes in the control program of visceral leishmaniasis in Brazil. Rev. Soc. Bras. Med. Trop. 34, 223-228.

Courtenay, O., Quinnell, R.J., Garcez, L.M., Shaw, J.J., Dye, C., 2002. Infectiousness of a cohort of braziliam dogs: why culling fails to control visceral leishmaniasis in areas of high transmission. J. Infect. Dis. 186, 1314-1320.

Coutinho, S.G., Nunes, M.P., Marzochi, M.C., Tramontano, N., 1985. A survey for American cutaneous and visceral leishmaniasis among $1342 \mathrm{dogs}$ from areas in Rio de Janeiro (Brazil) where the human diseases occur. Mem. Inst. Oswaldo Cruz. 80, 17-22.

Dietze, R., Barros, G.B., Teixeira, L., Harris, J., Michelson, K., Falqueto, A., Corey, R., 1997. Effect of eliminating seropositive canines on the transmission of visceral leishmaniasis in Brazil. Clin. Infect. Dis. 25, 1240-1242.

Dye, C., 1996. The logic of visceral leishmaniasis control. Am. J. Trop. Med. Hyg. 55, 125-130.

França-Silva, J.C., da Costa, R.T., Siqueira, A.M., Machado-Coelho, G.L.L., da Costa, C.A., Mayrink, W., Vieira, E.P., Costa, J.S., Genaro, O., Nascimento, E., 2003. Epidemiology of canine visceral leishmaniasis in the endemic area of Montes Claros municipally, Minas Gerais State, Brazil. Vet. Parasitol. 111, 161-173.

Gradoni, L., Pozio, E., Gramiccia, M., Maroli, M., Bettini, S., 1983. Leishmaniasis in Tuscany (Italy). VII. Studies on the role of the black Rattus rattus, in the epidemiology of visceral leishmaniasis. Am. J. Trop. Med. Hyg. 77 (4), 427-431. 
Gradoni, L., Gramiccia, M., Mancianti, F., Pieri, S., 1988. Studies on canine leishmaniasis control. 2. Effectiveness of control measures against canine leishmaniasis in the Isle of Elba, Italy. Trans. Roy. Soc. Trop. Med. Hyg. $82,568-571$.

Instituto Brasileiro de Geografia e Estatística (Ed.), 2001. Anuário Estatístico do Brasil-2000. Ministério Brasileiro do Planejamento e Orçamento, Rio de Janeiro, 288 pp.

Maurício, I.L., Howard, M.K., Stothard, J.R., Miles, M.A., 1999. Genomic diversity in the Leishmania donovani complex. Parasitology 119 (Pt 3), 237-246.

Moreira, E.D.J., Souza, V.M.M., Sreenivasan, M., Lopes, N.L., Barreto, R.B., Pontes-de-Carvalho, L.C., 2003. Peridomestic risk factors for canine leishmaniasis in urban dwellings: new findings from a prospective study in Brazil. Am. J. Trop. Med. Hyg. 69 (4), 393-397.

Nicolle, C., Comte, C., 1908. Origine Canine du Kala-azar. Bull. Soc. Pathol. Exe. 1908; i: 299-301.

Palatnik-de-Sousa, C.B., dos Santos, W.R., Franca-Silva, J.C., da Costa, R.T., Reis, A.B., Palatnik, M., Mayrink, W., Genaro, O., 2001. Impact of canine control on the epidemiology of canine and human visceral leishmaniasis in Brazil. Am. J. Trop. Med. Hyg. 65, 510-517.

Paranhos-Silva, M., Freitas, L.A., Santos, W.C., Grimaldi, G.J., Pontes-de-Carvalho, L.C., Oliveira-dos-Santos, A.J., 1996. A cross-sectional serodiagnostic survey of canine leishmaniasis due to Leishmania chagasi. Am. J. Trop. Med. Hyg. 55, 39-44.

Paranhos-Silva, M., Nascimento, E.G., Melro, M.C., Oliveira, G.G., dos Santos, W.L., Pontes-de-Carvalho, L.C., Oliveira-dos-Santos, A.J., 1998. Cohort study on canine emigration and Leishmania infection in an endemic area for American visceral leishmaniasis. Implications for the disease control. Acta Trop. 69, 75-83.

Tesh, R.B., 1995. Control of zoonotic visceral leishmaniasis: is it time to change strategies? Am. J. Trop. Med. Hyg. 52, 287-292.

Vieira, J.B., Coelho, G.E., 1998. Visceral leishmaniasis or kala-azar: the epidemiological and control aspects. Rev. Soc. Bras. Med. Trop. 31 (Suppl. 2), 85-92.

Zaffaroni, E., Rubaudo, L., Lanfranchi, P., Mignone, W., 1999. Epidemiological patterns of canine leishmaniasis (correction of leishmaniosis) in western Liguria (Italy). Vet. Parasitol. 81, 11-19. 